\title{
Belgeo
}

Revue belge de géographie

\section{Ségrégation socio-spatiale et ethnicisation des territoires scolaires à Marseille}

Socio-spatial segregation and ethnicization of schools, the case of Marseille (France)

\section{Gwenaëlle Audren et Virginie Baby-Collin}

\section{(2) OpenEdition}

Journals

\section{Édition électronique}

URL : https://journals.openedition.org/belgeo/18726

DOI : 10.4000/belgeo.18726

ISSN : 2294-9135

\section{Éditeur :}

National Committee of Geography of Belgium, Société Royale Belge de Géographie

\section{Référence électronique}

Gwenaëlle Audren et Virginie Baby-Collin, « Ségrégation socio-spatiale et ethnicisation des territoires scolaires à Marseille », Belgeo [En ligne], 2-3 | 2017, mis en ligne le 13 mars 2017, consulté le 28 juin 2022. URL : http://journals.openedition.org/belgeo/18726; DOI : https://doi.org/10.4000/belgeo. 18726

Ce document a été généré automatiquement le 29 septembre 2020.

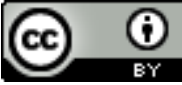

Belgeo est mis à disposition selon les termes de la licence Creative Commons Attribution 4.0 International. 


\section{Ségrégation socio-spatiale et ethnicisation des territoires scolaires à Marseille}

Socio-spatial segregation and ethnicization of schools, the case of Marseille

(France)

Gwenaëlle Audren et Virginie Baby-Collin

\section{Introduction}

1 L'ambition de l'école publique républicaine est de donner à tous les mêmes conditions d'accès à l'éducation. En France, le système du collège unique, mis en place en 1975, est censé procurer à tous les élèves la même offre scolaire jusqu'à l'âge de 15 ans environ ${ }^{1}$. La carte scolaire française, qui existe depuis 1963, définit des périmètres géographiques autour des établissements, assignant aux élèves une école selon leur adresse de résidence: ce sont les secteurs scolaires ${ }^{2}$. Ce système, qui permet de réguler les inscriptions et fonctionne selon des logiques de proximité, implique, dans des espaces urbains marqués par des processus de ségrégation socio-spatiale, le report de ces logiques ségrégatives dans l'enceinte de l'école: schématiquement, les élèves des quartiers bourgeois se retrouvent dans les établissements de leurs quartiers, à l'écart de ceux des quartiers populaires où se concentrent les élèves socialement défavorisés. Bien que les établissements de ces derniers soient dotés de moyens spécifiques (dans les réseaux d'éducation prioritaire), afin d'accompagner de façon plus suivie les publics défavorisés, ils pâtissent fréquemment d'une image dégradée, et obtiennent des résultats scolaires inférieurs à ceux des collèges des quartiers bourgeois. Alors que la loi d'orientation et de programmation pour la refondation de l'école de la république, votée en 2013, doit «veiller à la mixité sociale des publics scolarisés au sein des établissements d'enseignement $»^{3}$ et se donne pour objectif la réduction des inégalités scolaires, comment lutter contre les processus de ségrégation socio-spatiale à l'école? 
2 La carte scolaire est contournée de plusieurs manières : par la scolarisation dans les établissements privés, tout d'abord, qui échappent à toute sectorisation, et dont les inscriptions sont régulées directement par les chefs d'établissement; par l'obtention de dérogations pour certains motifs, gérées par les inspections académiques, ensuite ; enfin, par le jeu des options disponibles dans certains établissements, qui orientent vers eux les élèves demandeurs, même issus de secteurs différents. Les territoires scolaires, que nous définirons comme correspondant aux espaces de recrutement effectifs des établissements scolaires (élèves résidant dans le secteur de scolarisation, ou venus d'autres secteurs via le non-respect de la carte scolaire), sont ainsi issus de dynamiques complexes : ils sont d'abord le fruit des caractéristiques des secteurs scolaires définis selon des logiques de proximité autour des établissements (les caractéristiques sociales d'un quartier impliquant théoriquement celles du public de son collège); ils résultent aussi de l'offre scolaire spécifique à chaque établissement (définie à la fois par les politiques du Rectorat et des chefs d'établissement); ils sont enfin liés aux pratiques scolaires des familles (respect ou évitement des secteurs de la carte).

3 Si l'on sait que ces territoires sont socialement différenciés, comme l'ont montré un certain nombre d'études (Oberti, 2007 ; François et Poupeau, 2008 ; Merle, 2011, Oberti et al., 2012 ; Audren, 2015), on connait moins la place qu'y occupent les populations d'origine étrangère. En effet, en l'absence de toute statistique ethnique en France, les études relatives à la dimension ethnique des territoires sont difficiles à documenter de manière quantitative. Une étude menée auprès de plus de 300 collèges de l'académie de Bordeaux ${ }^{4}$ a mis en évidence une forme "d'apartheid scolaire, [...] non pas stricto sensu comme une volonté politique de séparation des "races", mais comme un ensemble de processus sociaux diffus conduisant à une ségrégation ethnique forte » (Felouzis, Liot \& Perroton, 2005, p. 16). D'autres travaux ont mis en évidence les processus d'ethnicisation à l'œuvre dans le milieu scolaire de façon plus qualitative (Dhume, 2011; Lorcerie, 2003 ; Payet, 2007). En accord avec la pensée constructiviste, l'ethnicisation renvoie au processus de construction de frontières et d'identification de groupes catégorisés selon leur origine ou leur culture, porteur de différenciation, d'infériorisation et d'inégalités (Barth, 1995; Bertheleu, 2007 ; De Rudder, Poirier \& Vourc'h, 2000 ; Primon, 2007).

4 Disposant de données nouvelles encore non exploitées (cf. infra), nous nous proposons ici d'interroger la place des populations d'origine étrangère dans les territoires scolaires, soit les processus d'ethnicisation, ou au contraire de mixité ethnique, à l'œuvre dans une grande ville française marquée à la fois par une forte ségrégation résidentielle, une forte différenciation scolaire, et une part importante de populations d'origine étrangère: Marseille. Nous mobilisons ici la notion d'ethnicisation pour questionner les processus qui conduisent à une surreprésentation des élèves d'origine étrangère dans certains établissements scolaires, par rapport à leur représentation dans l'espace urbain environnant.

Dans quelle mesure les processus ségrégatifs à l'œuvre dans l'espace urbain sont-ils reproduits dans les territoires scolaires ? La distribution spatiale des espaces de résidence des élèves d'origine étrangère se traduit-elle de la même manière dans les territoires scolaires des établissements? Si non, quels processus sont à l'œuvre dans la construction de ces territoires?

6 Nous exploitons la base de données SCORED du Rectorat de l'Académie d'Aix Marseille ${ }^{5}$, relative aux près de 40.000 collégiens résidant à Marseille en $2013^{6}$, mise en contexte par des données INSEE (recensement 2012, fichiers de la Direction Générale des Impôts de 
2011), et cartographiée à l'aide d'un système d'information géographique. L'analyse est nourrie de données qualitatives recueillies lors d'une enquête menée en 2015-16 auprès de chefs d'établissement, et d'enseignants de collèges marseillais, ainsi qu'auprès de plusieurs services académiques, même si nous nous restreignons ici notre analyse à celle des données quantitatives.

7 Après avoir introduit le contexte urbain et scolaire marseillais, caractérisé par de fortes inégalités socio-spatiales, nous analyserons les profils et la distribution spatiale des élèves d'origine étrangère, afin de questionner dans un dernier temps les logiques à l'œuvre dans la construction des territoires scolaires relatives à ce public spécifique, en nous appuyant sur quelques exemples significatifs éclairés par une analyse plus approfondie de deux collèges du centre-ville, qui, dans un contexte social proche, sont le théâtre de dynamiques très contrastées.

\section{Marseille, de fortes inégalités socio-spatiales, à la ville comme à l'école}

Qualifiée de «ville duale» (Donzel, 2005), Marseille se caractérise par une forte ségrégation socio-spatiale entre le sud de la ville, qui concentre les populations aisées, et le nord, où se regroupent de nombreux quartiers populaires, le centre-ville présentant la particularité d'être fortement peuplé par des groupes défavorisés. Ce tableau schématique comporte toutefois de nombreuses nuances, et d'importantes politiques urbaines de renouvellement, depuis les années 1990, ont contribué à modifier quelque peu cette géographie (Dorier et al., 2010 ; Donzel, 2014 ; Péraldi, Duport \& Samson, 2015). Ainsi, l'ouverture de nouveaux espaces à l'urbanisation au début des années 2000, dans les périphéries nord, est et sud, a favorisé dans des zones plus populaires le développement de lotissements destinés aux classes moyennes (souvent fermés et sécurisés); depuis 2003, la politique mise en place au niveau national par l'Agence nationale de la rénovation urbaine (ANRU) a impulsé une mixité sociale par le haut, pour attirer des populations de classes moyennes et supérieures dans des quartiers populaires (Dorier et al., 2012) ; enfin le centre-ville fait l'objet depuis 1995 d'une large opération d'intérêt national de restructuration appelée Euroméditerranée (Dubois, Olive, 2004; Berry-Chikhaoui, 2007 ; Bertoncello et Dubois, 2010), doublée d'une action municipale visant à redonner une attractivité à l'hypercentre par divers moyens (construction d'un tramway, rénovation des espaces publics, du vieux port, actions sur la rénovation du bâti dégradé). 
Figure 1. Géographie des revenus et collèges de l'éducation prioritaire à Marseille.

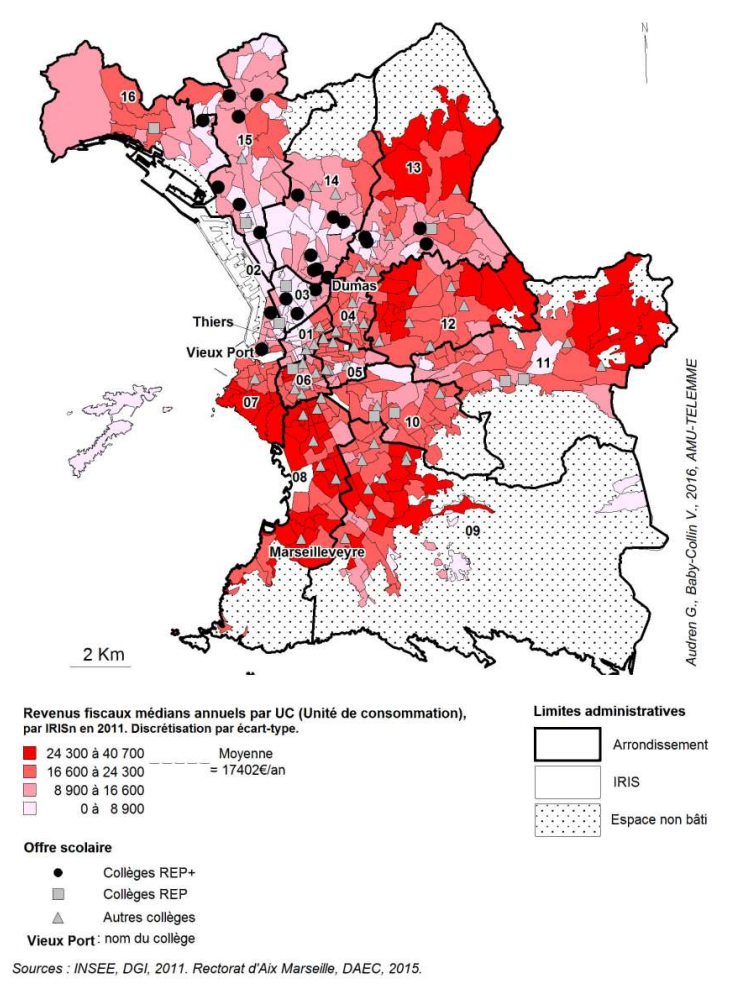

9 Avec $25 \%$ de pauvres ${ }^{7}$, Marseille a des revenus inférieurs à la moyenne française (en 2011, la moyenne des revenus y est de $17402 €$ / an contre $19218 €$ en France métropolitaine). Même si l'observation des revenus médians à l'échelle de l'IRIS ${ }^{8}$ - figure 1 - met en évidence une certaine hétérogénéité locale, l'échelle de l'arrondissement confirme les grandes tendances historiques de la ségrégation de l'espace marseillais (Roncayolo, 1996) : de $600 €$ dans le $3^{\text {ème }}$ arrondissement (centre-nord), où le taux de pauvreté atteint $55 \%$ de la population, à $2000 €$ dans le $8^{\text {ème }}$ arrondissement au sud, avec des IRIS atteignant pour les plus aisés $40670 €$, et des taux de pauvreté inférieurs à $10 \%$, similaires à ceux des arrondissements les plus riches de Lyon ou Paris (Maurin et Mazéry, 2014).

Conformément à de nombreuses études qui soulignent le lien entre ségrégation résidentielle et ségrégation scolaire (Oberti, 2007 ; Oberti et al., 2012 ; François et al., 2005 ; François et Poupeau, 2008; Merle, 2010), à Marseille, l'offre scolaire reflète les caractéristiques de la ségrégation socio-spatiale de la commune (Audren, Baby-Collin \& Dorier, 2016). Si l'on s'intéresse aux 87 collèges de la commune (scolarisant les élèves de 11 à 15 ans en moyenne, après l'école primaire et avant le lycée), 55 établissements publics, répartis sur l'ensemble du territoire, scolarisent $71 \%$ des collégiens, et 32 établissements privés, plus concentrés dans les zones centrales, accueillent $29 \%$ des près de 40000 collégiens marseillais (tableau 2). Parmi les établissements publics, 23 sur 55 appartiennent aux réseaux de l'éducation prioritaire (REP, voir figure 1 et tableau 2), attestant des difficultés sociales de plus de $40 \%$ des collèges de la ville (quand Paris et Lyon et Lille n'ont respectivement que 4 et 2 réseaux REP+). La définition de ces réseaux repose sur un indice social qui conjugue quatre variables : la proportion d'élèves issus de ZUS (zone urbaine sensible), le pourcentage d'élèves boursiers, d'élèves défavorisés ${ }^{9}$, le retard scolaire à l'entrée en sixième. La concentration de ces collèges dans les quartiers 
populaires du centre et du nord de la ville correspond à la géographie des quartiers populaires (figure 1). Dans les établissements privés du centre et du sud, et dans les collèges publics des quartiers sud, les caractéristiques sociales des établissements sont plus favorables (proportion élevée d'élèves favorisés, moins de retard scolaire et de boursiers).

\section{De la ségrégation socio-spatiale à la ségrégation ethnique au collège}

11 Après Paris, au même niveau que Lyon, Marseille est une ville d'immigration (tableau 1). Cette immigration de longue date a vu se succéder différentes origines au fil du temps, des Italiens de la fin du XIX ème siècle, aux Espagnols, Portugais, aux Algériens (qui représentent aujourd'hui environ le tiers de la population immigrée) et aux autres maghrébins après les indépendances coloniales. Plus récemment, l'immigration d'Afrique subsaharienne, comorienne, mais aussi issue des Balkans, d'Europe orientale, puis enfin du Proche et Moyen Orient, a diversifié le panorama (Temime, 2007). Les quartiers populaires du centre, à proximité de la gare et du vieux port, mais aussi ceux du nord de la commune, sont les plus marqués par cette présence, certains IRIS marseillais accueillant $40 \%$ de population immigrée et étrangère. Les logiques de cette géographie différenciée renvoient aux processus de ségrégation socio-spatiale ci-dessus identifiés.

Tableau 1. Etrangers / immigrés en 2012 (en \% de la population totale).

\begin{tabular}{|l|c|c|}
\hline & Population étrangere & Population immigrée \\
\hline France & $6 \%$ & $9 \%$ \\
\hline Paris & $15 \%$ & $20 \%$ \\
\hline Lyon & $8 \%$ & $12 \%$ \\
\hline Marseille & $8 \%$ & $13 \%$ \\
\hline
\end{tabular}

Source : Insee, recensement 2012

12 A partir de la base du SCORED 2013, nous avons identifié 9,2\% des collégiens marseillais (soit un effectif de 3670) comme étant d'origine étrangère, qu'ils soient de nationalité étrangère, ou nés à l'étranger ${ }^{10}$. Les plus nombreux sont originaires du Maghreb (42,5\%), les jeunes d'origine algérienne représentant à eux seuls le tiers de l'ensemble des collégiens d'origine étrangère, ce qui est conforme aux données disponibles pour l'ensemble de la population de Marseille, où l'on recense $35 \%$ d'Algériens parmi les origines étrangères (AgAM, 2015) ${ }^{11}$. Le second groupe le plus important est composé des collégiens originaires d'Afrique subsaharienne (19\%), dont une grande partie vient des Comores ( $9 \%$ du total des origines étrangères), un groupe d'immigration relativement récent (depuis les années 1980), et dans lequel le nombre d'enfants par famille est très élevé $^{12}$. Viennent ensuite les collégiens originaires du Proche et Moyen-Orient (10\%) groupe dans lequel nous avons inclus la Turquie, premier pays d'origine dans cet ensemble - puis d'autres pays européens (14\%), les élèves les plus nombreux en leur sein venant du Portugal, d'Italie et de Roumanie. Les collégiens venant d'Asie, des Amériques, ou de la Russie et des anciens pays de l'Union Soviétique sont les moins représentés (moins de $6 \%$ au total). 
13 La majorité des jeunes d'origine étrangère présente des indices plus élevés que la moyenne en termes de difficultés sociales et scolaires. Les catégories socioprofessionnelles de leurs parents font ressortir des élèves plus favorisés parmi ceux originaires d'Asie ou des Amériques (peu nombreux), alors que ceux d'origine maghrébine, d'Afrique subsaharienne, ou du Proche et Moyen-Orient, sont très majoritairement défavorisés (dépassant largement, près de deux fois plus, les $33 \%$ de collégiens marseillais défavorisés) et bénéficient pour la plupart de bourses attribuées sur critères sociaux. Ces collégiens sont aussi beaucoup plus nombreux à connaître un retard scolaire (un à deux ans de retard ou plus, pour plus de la moitié des collégiens originaires $\mathrm{du}$ Proche et Moyen-Orient par exemple $\left.{ }^{13}\right)$. Dans ce domaine, on peut évoquer les difficultés scolaires liées à la maîtrise de la langue française, qui n'est pas forcément la langue parlée à la maison dans les familles d'origine étrangère, qui peuvent générer un certain retard scolaire. Celui-ci est plus marqué pour les élèves de milieux défavorisés, majoritaires dans les groupes d'origine étrangère.

Figure 2. Catégories sociales des parents des collégiens marseillais selon leur origine (2013).

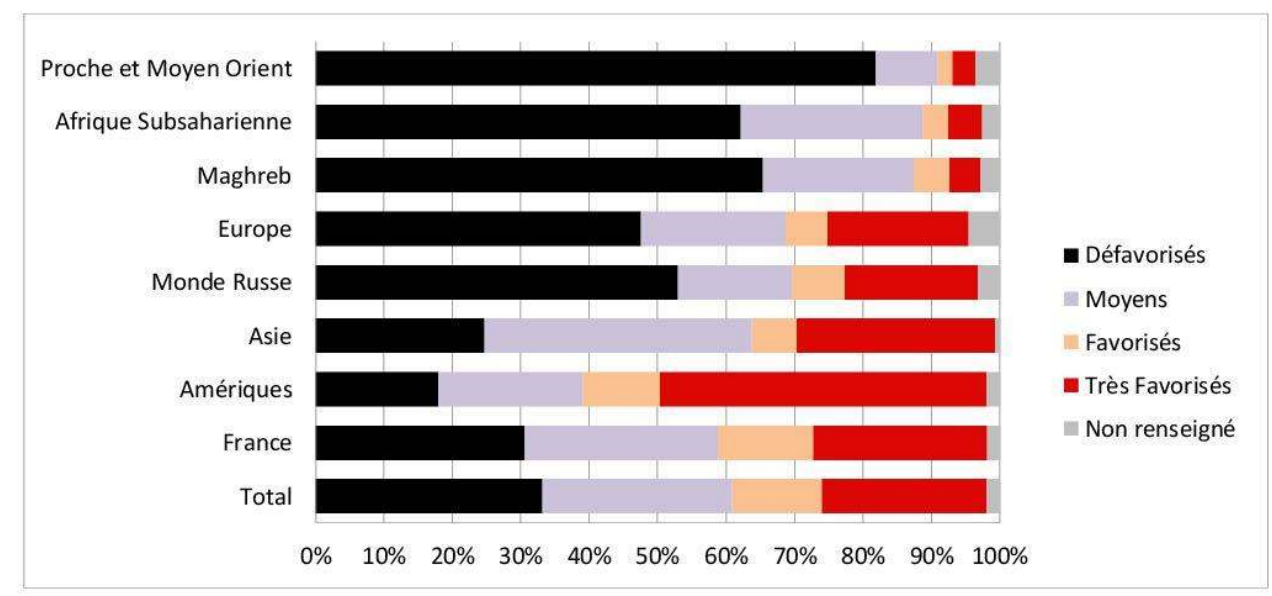

Source : Rectorat d'Aix Marseille, DAEC, 2013 
Figure 3. Espaces de résidence et de scolarisation des collégiens d'origine étrangère.

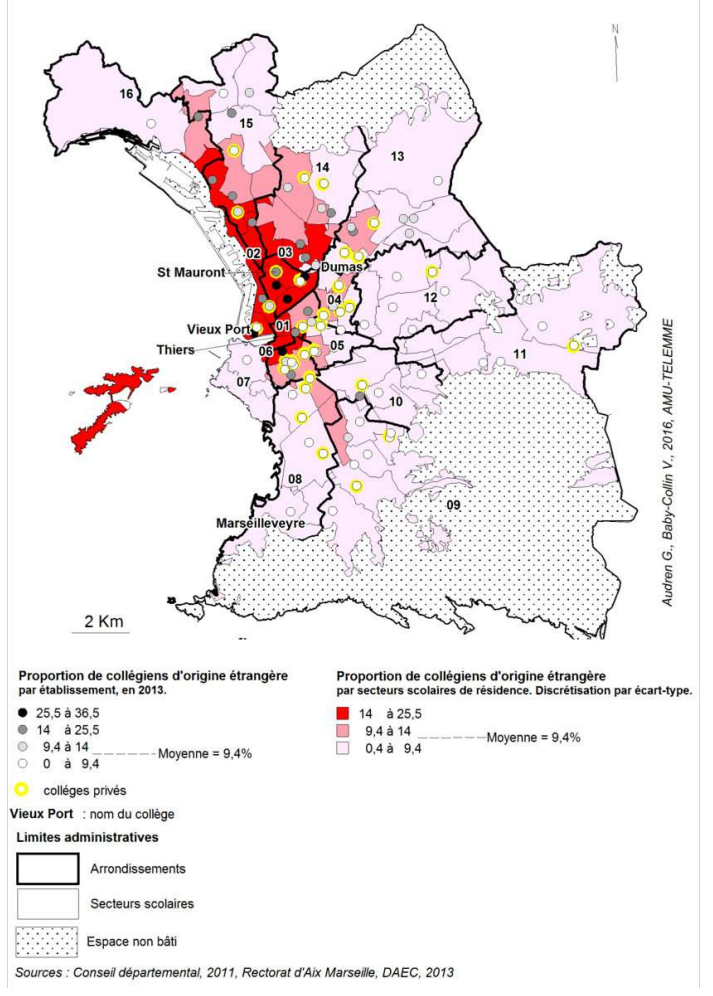

Les lieux de résidence des collégiens d'origine étrangère (figure 3) mettent en évidence une forte concentration dans les quartiers centraux ( $1^{\text {er }}, 2^{\text {ème }}$ et $3^{\text {ème }}$ arrondissements) et

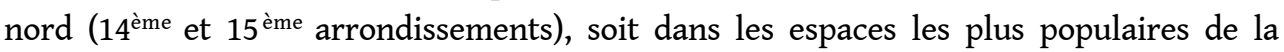
commune, bien que la géographie diffère quelque peu selon les groupes. La présence comorienne, proche et moyen-orientale est ainsi plus marquée dans les quartiers nord, autour des grandes cités de logements sociaux. La présence maghrébine est concentrée dans les arrondissements $\mathrm{du}$ centre et $\mathrm{du}$ nord de la commune. Plus favorisés socialement, les collégiens d'origine européenne habitent plutôt en situation centrale et dans les quartiers plus privilégiés du sud.

Les collégiens d'origine étrangère sont peu scolarisés dans le secteur privé ( $12 \%$ d'entre eux, contre près de $30 \%$ de l'ensemble des collégiens). Ils sont en revanche beaucoup plus représentés dans les établissements publics appartenant aux réseaux de l'éducation prioritaire (pour $60 \%$ d'entre eux, contre seulement $35 \%$ de l'ensemble des collégiens), ou encore dans les établissements bénéficiant de dispositifs d'accueil des élèves allophones, dits UPE $2 \mathrm{~A}^{14}$ - pour $53 \%$ d'entre eux, contre $28 \%$ de l'ensemble des collégiens $(\text { tableau } 2)^{15}$.

16 A l'échelle de la commune, 10 collèges (tous publics) seulement scolarisent le tiers des collégiens d'origine étrangère, et $13 \%$ de l'ensemble des collégiens. Ils accueillent $61 \%$ de catégories défavorisées et seulement $7 \%$ de très favorisés ; à l'opposé, 58 collèges (dont la totalité des 32 collèges privés de la commune) scolarisent un tiers des collégiens d'origine étrangère, et $61 \%$ de l'ensemble des collégiens, accueillant en moyenne $24 \%$ de catégories défavorisées et $28 \%$ de très favorisés. Ces résultats soulignent, de manière encore plus prononcée que dans l'étude menée dans l'académie de Bordeaux il y a une dizaine d'années, la force des processus de ségrégation sociale et ethnique au collège ${ }^{16}$. 
Tableau 2. Les collégiens par type d'établissement en 2013.

\begin{tabular}{|c|c|c|c|}
\hline Rubriques (numéro et description) & Collège Thiers & Collège Vieux Port & Marseille* \\
\hline I-Nombre d'élèves dans le secteur scolaire & 279 & 378 & 39894 \\
\hline Nombre d'élèves dans l'établissement & 435 & 324 & 39694 \\
\hline & & & -200 \\
\hline $\begin{array}{l}\text { II-Taux d'évitement (\% des élèves du secteur } \\
\text { qui ne sont pas scolarisés dans le collège de }\end{array}$ & 31 & 59 & 50 \\
\hline & 44 & 27 & 42 \\
\hline - issus de catégories favorisées** & 21 & 35 & 28 \\
\hline -issus de catégories moyennes & 31 & 34 & 28 \\
\hline $\begin{array}{l}\text { - issus de catégories défavorisées } \\
\text {-d'origine étrangère }\end{array}$ & 22 & 18 & 8 \\
\hline III-Taux d'attractivité (\% des élèves de & 56 & 52 & 29 \\
\hline 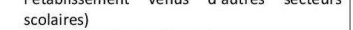 & 53 & 5 & 42 \\
\hline - issus de catégories favorisées & 24 & 20 & 28 \\
\hline - issus de catégories moyennes & 21 & 69 & 28 \\
\hline $\begin{array}{l}\text { - issus de catégories défavorisées } \\
\text { - d’origine étrangère }\end{array}$ & 17 & 42 & 8 \\
\hline IV-\% de boursiers & & & \\
\hline - dans le secteur & 36 & 43 & 34 \\
\hline - dans l'établissement & 42 & 78 & 34 \\
\hline - venus d'autres secteurs (attractivité) & 42 & 80 & 28 \\
\hline V-\% d'élèves en retard scolaire & 12 & 45 & 20 \\
\hline $\mathrm{Vl}$ \% d'élèves d'origine étrangère & & & \\
\hline - dans le secteur & 16.5 & 23 & 9.5 \\
\hline - dans l'établissement & 16 & 36 & 9.5 \\
\hline - venus d’autres secteurs (attractivité) & 17 & 42 & 8.3 \\
\hline $\begin{array}{l}\text { - Nombre de nationalités représentées dans } \\
\text { l'établissement }\end{array}$ & 22 & 31 & $\begin{array}{c}14 \\
\text { (moyenne) }\end{array}$ \\
\hline $\begin{array}{l}\text { VII- \% d'élèves scolarisés à l'étranger l'année } \\
\text { précédente (2012) }\end{array}$ & & & \\
\hline -dans le secteur & 2.9 & & 0.5 \\
\hline - dans l'établissement & 0.7 & 4.3 & 0.5 \\
\hline VIII- \% d'élèves favorisés & & & \\
\hline - dans le secteur & 44 & 22 & 37 \\
\hline - dans l'établissement & 49 & 10 & 37 \\
\hline - venus d'autres secteurs (attractivité) & 53 & 5 & 42 \\
\hline IX-\% d'élèves défavorisés & & & \\
\hline - dans le secteur & 27 & 42 & 33 \\
\hline - dans Pétablissement & 23 & 61 & 33 \\
\hline - venus d'autres secteurs (attractivité) & 21 & 69 & 28 \\
\hline $\begin{array}{l}\text { X-Taux de réussite au brevet des collèges } \\
\text { (2016) }\end{array}$ & 82 & 68 & $83^{* * *}$ \\
\hline - dont avec mentions ( $>12 / 20$ de moyenne) & 61 & 29 & $54^{* * *}$ \\
\hline $\begin{array}{cccc}\text { Xl-Sections } & \text { spécifiques } & \text { dans } & \text { les } \\
\text { établissements } & \end{array}$ & $\begin{array}{c}\text { Musique, classe } \\
\text { européenne }\end{array}$ & 2 classes UPE2A & \\
\hline
\end{tabular}

*Pour la colonne Marseille, qui représente les données des 39894 collégiens résidant dans la commune, les chiffres par secteur scolaire et par établissement sont pour l'essentiel identiques, dans la mesure où il s'agit de la moyenne pour toute la commune (soit tous les secteurs et tous les établissements). L'écart de 200 élèves à l'échelle de la ville (rubrique I) correspond à des collégiens scolarisés dans des communes voisines. En revanche, les taux d'attractivité et d'évitement (rubrique II et III) diffèrent dans leur valeur générale, car l'évitement prend en compte les départs également vers les établissements privés (non sectorisés), quand l'attractivité ne rend compte que des établissements publics (sectorisés).

** On a regroupé ici les élèves issus des catégories favorisées et très favorisées telles que proposées par les données du Rectorat (cf. supra). Le total des élèves des catégories favorisées, moyennes et défavorisées est égal à $100 \%$ (rubriques II et III).

*** Établissements de l'ensemble du département des Bouches du Rhône

Source : SCORED 2013, DAEC, Rectorat d'Aix-Marseille. Pour les résultats au brevet 2016 : www.france-examens.com

17 La superposition des données relatives aux secteurs scolaires de résidence des collégiens d'origine étrangère (en à-plats sur la figure 3) et de celles relatives à leurs établissements de scolarisation (en cercles dégradés de gris) met en évidence plusieurs phénomènes.

D'abord, elle confirme l'adéquation entre les espaces de résidence principaux des collégiens d'origine étrangère (quartiers centraux et nord), les zones de plus faibles revenus et les établissements de l'éducation prioritaire (identifiés sur la figure 1) : c'est dans les zones les plus populaires que les collégiens d'origine étrangère sont les plus nombreux à résider et à être scolarisés ${ }^{17}$. C'est dans ces zones que se concentrent aussi les établissements de l'éducation prioritaire. Les collégiens d'origine étrangère sont en effet relativement moins nombreux, tant dans les secteurs scolaires que dans les établissements, au sud et à l'est de la commune, dans les quartiers socialement plus privilégiés. 
19 Ensuite, la carte confirme que les établissements privés (entourés d'un cercle jaune) scolarisent relativement peu de collégiens d'origine étrangère, même par rapport à leur proportion moyenne dans les quartiers qui les entourent, à quelques exceptions près : ainsi le collège privé catholique St-Mauront, situé dans l'arrondissement le plus pauvre de la commune (3 $\left.3^{\mathrm{èm}}\right)$, l'un des plus marqués par la présence immigrée, scolarise $25 \%$ de collégiens d'origine étrangère ${ }^{18}$.

Enfin, et c'est sans doute le résultat le plus intéressant, on observe une plus forte concentration des élèves d'origine étrangère dans certains établissements que ne le laisserait attendre la réalité de leur concentration dans les espaces de résidence. En effet, alors que l'on ne trouve pas plus de $25 \%$ de collégiens d'origine étrangère dans les secteurs scolaires où ils sont le plus concentrés (dans le centre-ville $-1^{\text {er }}, 2^{\text {ème }}$ et $3^{\text {ème }}$ arrondissements - et certains secteurs des 14 et $15^{\text {ème }}$ arrondissements au nord), le taux de concentration des collégiens d'origine étrangère atteint $36,5 \%$ dans le collège où ils sont proportionnellement les plus nombreux (collège du Vieux Port, en centre-ville); et cinq collèges, tous situés dans le centre- ville, concentrent plus de $25 \%$ de collégiens d'origine étrangère.

21 Ces résultats montrent donc que la scolarisation contribue à concentrer les élèves d'origine étrangère et à renforcer les processus ségrégatifs observés dans la commune : les territoires scolaires, c'est-à-dire les espaces de recrutement effectifs (et non théoriques, ce que sont les secteurs) des établissements scolaires, apparaissent ainsi comme plus ethniquement ségrégués que les quartiers (ou les secteurs scolaires) dans lesquels se situent les collèges. Comment expliquer cette ethnicisation des territoires scolaires, ou la surreprésentation des élèves d'origine étrangère dans les établissements, par rapport à celle de leur secteur de recrutement?

\section{L'ethnicisation des territoires scolaires : entre pratiques de non-respect des familles et structuration d'une offre inégalement attractive}

Pour comprendre les mécanismes en jeu, il importe de revenir sur les logiques à l'œuvre dans le non-respect de la carte scolaire et dans l'attractivité différenciée des collèges. Un zoom sur deux établissements publics contrastés du centre-ville, situés à moins de $2 \mathrm{~km}$ l'un de l'autre, mis en perspective avec les tendances générales de la commune, nous permet de les éclairer (tableau 3).

Le choix de ces deux établissements est justifié par leur proximité géographique, ainsi que par leur situation dans le centre-ville, c'est-à-dire à la fois un espace plutôt populaire mais affecté par des dynamiques de gentrification, et un espace dans lequel la présence des étrangers et immigrés est forte ${ }^{19}$. Il se justifie aussi par les représentations et les publics contrastés de ces établissements très proches, qui interrogent sur les processus à l'œuvre dans la construction des publics scolaires. Le lycée Thiers, situé dans le quartier populaire de Noailles ( $1^{\mathrm{er}}$ arrondissement), est l'un des établissements cotés de Marseille. Selon ses résultats, c'est le meilleur lycée public de la ville, et il dispose de très bonnes classes préparatoires pour les grandes écoles, après le baccalauréat. Son collège est en conséquence assez prisé, considéré comme une voie d'accès au lycée, mais il est situé dans un secteur scolaire populaire du centre, avec de nombreux élèves d'origine étrangère ( $16,5 \%$ selon les données du Rectorat - tableau 3). Le collège Vieux Port, dans le quartier 
populaire historique du Panier, à deux pas du vieux port ( $2^{\text {ème }}$ arrondissement), est quant à lui un simple collège (sans lycée associé), aux résultats scolaires médiocres (résultats au brevet des collèges bien inférieurs à la moyenne départementale), situé dans un environnement encore plus populaire que Thiers ( $42 \%$ d'élèves défavorisés dans le secteur - $33 \%$ à Marseille et $27 \%$ dans le secteur de Thiers), et très marqué par la présence étrangère.

Tableau 3. Indicateurs relatifs à la construction des territoires scolaires.

\begin{tabular}{|l|c|c|c|c|c|}
\hline & \multicolumn{4}{|c|}{ Collèges publics } & Collèges privés \\
\cline { 2 - 6 } & $\begin{array}{c}\text { Tous collèges } \\
\text { publics }\end{array}$ & $\begin{array}{c}\text { Collèges } \\
\text { REP+ }\end{array}$ & $\begin{array}{c}\text { Collèges } \\
\text { éducation } \\
\text { prioritaire }\end{array}$ & $\begin{array}{c}\text { Collèges avec } \\
\text { UPE2A }\end{array}$ & \\
\hline $\begin{array}{l}\text { \% de l'ensemble } \\
\text { des collégiens }\end{array}$ & $\mathbf{7 1}$ & 26 & 35 & 28 & $\mathbf{2 9}$ \\
\hline $\begin{array}{l}\text { \% de l'ensemble } \\
\text { des collégiens } \\
\text { d'origine étrangère }\end{array}$ & $\mathbf{8 8}$ & 49 & 60 & 54 & $\mathbf{1 2}$ \\
\hline
\end{tabular}
scolarise plus d'élèves que n'en contient son secteur $(+156)$, quand Vieux Port en scolarise moins (-54). La différence entre les profils des collégiens du secteur, ceux effectivement scolarisés dans les établissements, et ceux qui viennent d'autres secteurs, met en évidence des processus de spécialisation sociale et ethnique. A Thiers, il y a davantage d'élèves favorisés parmi les collégiens venus d'autres secteurs (53\%) que parmi ceux du secteur (44\%), ce qui conduit à relever la part des publics favorisés dans le collège (49\%). C'est le contraire qui se produit au Vieux Port, où $61 \%$ des collégiens sont de profil défavorisé, contre seulement $42 \%$ dans le secteur (rubrique IX).

L'écart entre le taux d'élèves d'origine étrangère résidant dans le secteur du collège Vieux Port ( $23 \%)$, et effectivement scolarisé dans l'établissement (36,5\%), atteint 13 points, attestant d'un renforcement au sein de l'école de la ségrégation ethnique (rubrique VI). A contrario, le collège Thiers scolarise moins d'élèves d'origine étrangère (16\%) que ne le laisserait attendre la réalité de son secteur scolaire $(16,5 \%)$ : son territoire scolaire effectif est ainsi légèrement moins ethnicisé que le quartier.

L'évitement, ou le non-respect de la carte scolaire ${ }^{20}$, est à Marseille très élevé, concernant un élève sur deux (rubrique II). Le profil général de ces élèves est socialement marqué : $42 \%$ de profils favorisés, seulement $28 \%$ de profils défavorisés. La majorité d'entre eux ont recours au secteur privé (57 \%), mais ils sont plus nombreux à faire ce choix parmi les catégories favorisées $(72 \%)$ et moyennes (61\%). A l'inverse, les collégiens défavorisés qui ne sont pas scolarisés dans leur secteur sont plutôt inscrits dans d'autres établissements publics (66\%). Le coût représenté par une inscription dans un établissement privé est un des éléments d'explication, avec la sélectivité propre des établissements, ainsi que les conditions d'accès (une grande part des établissements privés se trouve dans les quartiers résidentiels aisés de la commune, et sont donc moins facilement accessibles aux catégories populaires qui y résident peu). Ces données confirment les résultats de nombreuses études, selon lesquelles les catégories les plus favorisées ont les pratiques d'évitement de la carte scolaire les plus actives, et les plus tournées vers les établissements privés (Van Zanten, 2001; Oberti, 2007; François et Poupeau, 2008; Lehman-Frisch, 2009 ; Audren, 2015 ; Audren, Dorier \& Rouquier, 2016). 
Les collégiens d'origine étrangère, au profil social plutôt défavorisé, ont moins recours au non-respect que l'ensemble des élèves; ils représentent $8 \%$ de ceux qui ne respectent pas la carte scolaire à Marseille, alors qu'ils sont plus de $9 \%$ de l'effectif total (rubrique VI). Ce sont toutefois $44 \%$ des collégiens d'origine étrangère qui ne sont pas scolarisés dans leur secteur. Seuls $28 \%$ d'entre eux sont scolarisés dans les établissements privés, mais les trois quarts de ceux qui y ont recours appartiennent aux catégories moyennes et favorisées. Cette forme d'évitement est plus marquée pour les collégiens originaires d'Amérique ou d'Asie (plus de la moitié des évitants), dans des collèges de bonne réputation du centre-ville ou du $8^{\text {ème }}$ arrondissement, que pour les collégiens originaires d'Afrique subsaharienne ou du Maghreb, dont seulement le quart des évitants sont inscrits dans des collèges privés, plutôt des établissements de proximité, pas forcément connus pour la qualité de leurs résultats scolaires, particulièrement dans le centre-ville ou les quartiers nord.

L'évitement vers le privé, forme majoritaire du non-respect de la carte scolaire, ne concerne ainsi qu'une minorité des collégiens d'origine étrangère: pour $72 \%$ des collégiens d'origine étrangère évitants, et $43 \%$ de l'ensemble des collégiens évitants, les logiques de non-respect se jouent entre établissements publics. L'attractivité des collèges, qui mesure la part des élèves scolarisés dans les collèges publics qui n'habitent pas le secteur de recrutement (rubrique III, tableau 3), en moyenne de $29 \%$ à l'échelle de la commune, est ainsi fort variable selon les établissements (de 8 à $59 \%$ ).

Elle est liée à deux principaux mécanismes. D'abord, l'obtention de dérogations par les familles qui permet sous certaines conditions de déroger à son secteur scolaire ${ }^{21}$. Ensuite, l'affectation des élèves dans des collèges disposant d'une offre spécifique non disponible dans leur secteur, telles les sections de langues rares, les sections européennes, sportives ou musicales, les SEGPA ${ }^{22}$, ou encore les dispositifs UPE2A, spécifiquement dédiés aux élèves étrangers nouvellement arrivés en France, disponibles dans 23 collèges marseillais.

Parmi les collèges très attractifs, on trouve ainsi aussi bien des établissements de très bonne réputation, aux très bons résultats scolaires, comme le collège Thiers, dans le centre-ville, ou Marseilleveyre, dans les quartiers aisés du sud de la ville, qui disposent de sections spécifiques prisées par les populations aisées (sections européennes, sportives, musicales), que des établissements relevant de réseaux de l'éducation prioritaire, aux résultats scolaires faibles, et réputés peu attractifs, comme le collège Alexandre Dumas dans le $14^{\text {ème }}$ arrondissement au nord (dont le tiers de la population scolaire est composé d'élèves inscrits dans des sections SEGPA, venus donc de différents secteurs scolaires), ou le collège du Vieux Port, qui dispose de deux dispositifs UPE2A, et dont plus de la moitié des élèves sont extérieurs au secteur (attractivité de $52 \%$, presque autant qu'à Thiers).

31 Si les logiques de l'affectation en section bilangue, européenne, musicale ou sportive, relèvent généralement des choix stratégiques des familles, pour permettre à leurs enfants de suivre des cursus privilégiés au sein des établissements scolaires (ce qui est le cas en général à Thiers, qui dispose de sections musicales et internationales), d'autres affectations sont plutôt subies, et relèvent de formes d'orientation guidées par l'éducation nationale. Il en est ainsi des élèves scolarisés en dispositif UPE2A ou section SEGPA, vers lesquelles les jeunes d'origine étrangère sont orientés plus souvent que les autres, comme l'ont montré plusieurs études (Zaffran, 2010 ; Felouzis, 2003) ; à Marseille, $9 \%$ des collégiens d'origine étrangère qui ne respectent pas la carte scolaire sont inscrits en SEGPA. 

dispositif UPE2A, spécifique à la scolarisation différenciée des élèves allophones nouvellement arrivés en France, et conçu comme une passerelle devant permettre aux élèves de rejoindre les classes ordinaires au terme d'une (exceptionnellement deux) années de scolarisation dans l'établissement ${ }^{23}$. Le collège Vieux Port est le seul établissement de la commune qui accueille deux classes UPE2A, ce qui explique en partie l'ethnicisation de son territoire scolaire, pour les collégiens nouvellement arrivés ${ }^{24}: s^{\prime} i l s$ sont $2 \%$ à résider dans le secteur du Vieux Port, le double y est effectivement scolarisé $(4,3 \%)$; a contrario, ils sont près de $3 \%$ des collégiens dépendant du secteur Thiers, mais seulement $0,7 \%$ à y être réellement scolarisés. L'établissement n'accueillant pas de dispositif UPE2A, les élèves sont orientés vers d'autres collèges. On peut parler ici d'une politique conduisant à l'ethnicisation des territoires scolaires, ou de construction institutionnelle de l'ethnicité (Lorcerie, 2003 ; Bertheleu, 2007).

\section{Conclusion}

Dans un contexte urbain ségrégué, la carte scolaire française tend à reporter les inégalités sociales et ethniques des quartiers sur les établissements scolaires. Ainsi à Marseille, les collèges d'éducation prioritaire, ou disposant de sections d'accueil pour les jeunes allophones, sont concentrés principalement dans les arrondissements populaires du nord et du centre, où se concentrent aussi les plus gros effectifs de jeunes d'origine étrangère.

La carte scolaire est toutefois à Marseille contournée par la moitié des collégiens, par des mécanismes d'évitement vers le privé, d'obtention de dérogations, ou d'affectation en fonction d'options spécifiques. L'écart entre les profils des secteurs scolaires définis par l'administration et les territoires scolaires réels des établissements montre que ces contournements renforcent les inégalités scolaires: d'une part en renforçant la spécialisation sociale des collèges, d'autre part en en accentuant l'ethnicisation, c'est-àdire la concentration des élèves d'origine étrangère dans un petit nombre d'établissements, qui cumulent généralement difficultés sociales et retards scolaires.

En 2007, l'assouplissement de la carte scolaire devait favoriser la mobilité scolaire des publics peu favorisés, les boursiers devenant par exemple prioritaires dans les demandes de dérogation. Nos observations convergent avec les travaux qui constatent que cette politique, pensée pour améliorer la mixité scolaire, a plutôt contribué à l'accentuation de la ségrégation par le renforcement de l'évitement des collèges publics de la part des familles plus favorisées en direction d'établissements privés ; les catégories défavorisées, et à Marseille, les collégiens d'origine étrangère, demeurant les plus captifs dans l'espace scolaire.

Les politiques d'options et de sections proposées par les établissements jouent aussi un grand rôle dans le renforcement des inégalités des territoires scolaires. Dans ce domaine, le rôle des instances académiques, pour réguler et structurer une offre scolaire qui tienne compte des spécificités des territoires mais aussi des objectifs de mixité scolaire, devrait être de pouvoir agir non pas dans le sens d'un renforcement des spécialisations sociales ou ethniques des établissements, mais dans celui d'une répartition plus juste et moins inégalitaire des élèves dans les établissements publics. 


\section{BIBLIOGRAPHIE}

AGAM (2015), "Qui sont les Marseillais ? Radioscopie des habitants », Regards de l'agence

d'urbanisme de l'agglomération de Marseille, 28.

AUDREN G. (2015), Géographie de la fragmentation urbaine et territoires scolaires à Marseille, thèse de doctorat, Université d'Aix-Marseille.

AUDREN G. DORIER E. \& ROUQUIER D. (2016), Géographie de la fragmentation urbaine et territoire scolaire : effets des contextes locaux sur les pratiques scolaires à Marseille, Rennes, ESO, CREAD, Université de Rennes 2, http://services.univ-rennes2.fr/reso/outils/INEDUC/index.php.

AUDREN G., BABY-COLLIN V. \& DORIER E. (2016), « Quelles mixités dans une ville fragmentée? Une approche par les dynamiques locales de l'espace scolaire à Marseille », Lien social et Politiques, Montréal, pp. 38-61.

BARTH F. (1995 (1969)), « Les groupes ethniques et leurs frontières », in POUTIGNAT P. \& STREIFF-FENART J. (éd.), Théories de l'ethnicité, Paris, PUF, pp. 203-249.

BEN AYED C., BROCCOLICHI S. \& MONFROY B. (2013), « Quels impacts de l'assouplissement de la carte scolaire sur la ségrégation sociale au collège ? Tendances nationales et déclinaisons locales contrastées ", Éducation et formations, 83, pp. 39-57.

BERRY CHIKHAOUI I. (2007), « Les citadins face aux enjeux d'internationalisation de la ville. Casablanca et Marseille, où est le nord, où est le sud ? ", Autrepart, 1, 41, pp. 149-163.

BERTHELEU H. (2007), " Sens et usages de "l'ethnicisation" ", Revue européenne des migrations internationales, $23,2$.

BERTONCELLO B., DUBOIS J. (2010), Marseille Euroméditerranée. Accélérateur de métropole, Marseille, Parenthèses.

DHUME F. (2011) Entre l'école et l'entreprise, la discrimination ethnico-raciale dans les stages. Une sociologie publique de l'ethnicisation des frontières et de l'ordre scolaires, thèse de doctorat, Université de Provence, Aix Marseille I.

DONZEL A. (2005), « Marseille une métropole duale », Faire savoirs, 5, pp. 13-19.

DONZEL A. (2014), Le nouvel esprit de Marseille, Paris, L'Harmattan, Questions contemporaines/ Série questions urbaines.

DORIER APPRILL E. (dir.), BERRY-CHIKHAOUI I., BRIDIER S., BABY-COLLIN V., AUDREN G. \& GARNIAUX J. (2010), La diffusion des ensembles résidentiels fermés à Marseille. Les urbanités d'une ville fragmentée, Paris, rapport de recherche PUCA.

DORIER E., BERRY-CHIKHAOUI I. \& BRIDIER S. (2012), « Fermeture résidentielle et politiques urbaines, le cas marseillais », Articulo - Journal of Urban Research, 8, http:// articulo.revues.org/1973.

DUBOIS J., OLIVE M. (2004), « Euroméditerranée, négociations à tous les étages. Etat, promoteurs et propriétaires dans une ville en crise ", Les Annales de la recherche urbaine, 97, pp. 103-111.

FELOUZIS G. (2003), « La ségrégation ethnique au collège et ses conséquences », Revue française de sociologie, 44, pp. 413-447. 
FELOUZIS G., LIOT F. \& PERROTON J. (2005), L'apartheid scolaire. Enquête sur la ségrégation ethnique dans les collèges, Paris, Seuil.

FRANÇOIS J.C., POUPEAU F. (2008), Le sens du placement. Ségrégation résidentielle et ségrégation scolaire, Paris, Raisons d'agir.

INSPECTION GENERALE DE L'ÉDUCATION NATIONALE (2013), Conséquences des mesures d'assouplissement de la carte scolaire après 2007, Paris, 98 p.

LEHMAN FRISCH S. (2009), «La ségrégation : une injustice spatiale ? Questions de recherche », Annales de Géographie, Paris, Armand Colin, pp. 94-115.

LORCERIE F. (2003), L'école et le défi ethnique, ESF Editeur, Collection Actions Sociales/ confrontations.

MAURIN L., MAZERY V. (2014), « Les taux de pauvreté des 100 plus grandes communes de France ", Compas études, 11, $4 \mathrm{p}$.

MERLE P. (2011), « La carte scolaire et son assouplissement, Politique de mixité sociale ou de ghettoïsation des établissements? ", Sociologie, 1, pp. 39-51.

OBERTI M. (2007), L'école dans la ville : ségrégation, mixité carte scolaire, Paris, Presses de Sciences Politiques.

OBERTI M., PRETECEILLE E. \& RIVIERE C. (2012), Les effets de l'assouplissement de la carte scolaire dans la banlieue parisienne, Rapport intermédiaire HALDE/DEPP, Paris, Sciences Po-OSC.

PAYET J.P. (2007) L'« ethnicisation » des rapports scolaires et la discrimination ethnique au sein de l'école : un bilan des travaux en France et en Europe, Site du Laboratoire CNRS GRS.

PERALDI M., DUPORT C. \& SAMSON M. (2015), Sociologie de Marseille, Paris, La Découverte, coll. Repères Sociologie.

PRIMON J.L. (coord.) (2007), « L'ethnicisation et la racisation des rapports sociaux en question », Faire Savoirs, 7.

RONCAYOLO M. (1996), Les grammaires d'une ville. Essai sur la genèse des structures urbaines à Marseille, Paris, EHESS.

RUDDER (de) V., POIRET C. \& VOURC'H F. (2000), L'inégalité raciste. L'universalité républicaine à l'épreuve, Paris, PUF.

TEMIME E. (2007), Migrance. Histoire des migrations à Marseille, Marseille, Jeanne Laffitte, 4 vol.

VAN ZANTEN A. (2001), L'école de la périphérie : scolarité et ségrégation en banlieue, Paris, PUF.

ZAFFRAN J. (2010), « Entrer en Segpa et en sortir ou la question des inégalités transposées », La Documentation française, Formation emploi, 109, pp. 85-97, http://formationemploi.revues.org/2804.

\section{NOTES}

1. Quand d'autres pays européens (par exemple l'Allemagne) ont fait le choix d'une éducation différenciée dès la fin du cycle primaire.

2. Ils sont pour l'essentiel définis selon des logiques de proximité géographique et d'accessibilité (transports en commun), tout en respectant les équilibres de peuplement par rapport aux capacités d'accueil des établissements. Conformément au code de l'éducation, la délimitation des secteurs scolaires relève de la responsabilité des conseils départementaux, qui ont en charge la gestion des collèges en France (celle des écoles primaires relevant des municipalités). 
3. Article 2 de la LOI n²013-595, publiée au JORF n0157 du 9 juillet 2013, p. 11379.

4. En l'absence de données sur les origines des élèves, les chercheurs ont construit leur analyse à partir d'un classement des prénoms des élèves, identifiés selon leurs origines.

5. Dans le cadre d'une convention de collaboration de recherche établie avec le Rectorat. En France, le Rectorat regroupe les services déconcentrés de l'Etat relatifs à l'administration de l'éducation nationale, placés sous la direction du Recteur, au sein du périmètre de l'académie, circonscription administrative de référence pour l'éducation nationale (30 en France). Les auteures remercient ici MM. Chénier et Chauvot de la Direction de l'Analyse, des Etudes et de la Communication (DAEC) du Rectorat pour leur collaboration.

6. Ensemble de variables socio-économiques relatives aux familles (CSP, nombre d'enfants dans la fratrie), aux élèves (lieu de naissance et nationalité, âge, lieu de résidence et établissement de scolarisation, type de classe, scolarisation antérieure, statut scolaire - boursier, retard scolaire, options...).

7. La pauvreté est définie par le taux de ménages qui perçoivent moins de $60 \%$ du revenu médian national.

8. Îlot regroupé pour l'information statistique, la plus petite unité spatiale statistique en France, d'environ 2000 habitants en moyenne.

9. Le ministère de l'Éducation Nationale classe les métiers des responsables légaux des élèves en cinq classes principales : très favorisés (cadres supérieurs, enseignants, patrons d'entreprises de 10 salariés et plus, professions libérales), favorisés (professions intermédiaires : de la santé, du travail social, de la fonction publique, et administratives, techniciens, contremaîtres, agents de maîtrise), moyens (cadres moyens du public et du privé, employés, artisans, commerçants et agriculteurs), défavorisés (ouvriers et inactifs) et non renseignés (information inconnue ou non renseignée). L'originalité par rapport aux regroupements de l'INSEE est la place accordée au capital culturel (les enseignants sont classés dans les très favorisés).

10. En l'absence de données relatives aux origines des parents des élèves, et à partir des données dont nous disposons dans la base SCORED (pays de naissance et nationalité des élèves), nous qualifions ici les élèves d'origine étrangère comme étant à la fois les élèves nés à l'étranger, quelle que soit leur nationalité actuelle (française ou étrangère), et les élèves de nationalité étrangère nés en France. Nous ne cernons ainsi pas les élèves français nés en France, issus de parents immigrés. Sont inclus en revanche les quelques élèves français nés à l'étranger dans des familles expatriées, sans origine étrangère à proprement parler, groupe estimé comme étant très résiduel. Nous disposons pour chaque élève de sa nationalité et de son pays de naissance ; pour l'analyse, nous avons regroupé les collégiens en plusieurs groupes d'origines étrangères, selon des logiques de proximité géographique et culturelle : Maghreb (du Maroc à l'Egypte), Afrique subsaharienne, Proche et Moyen Orient (incluant ici la Turquie), Europe, monde russe (Russie et territoires de l'ancienne Union soviétique), Asie-Océanie, Amériques.

11. L'étude Qui sont les marseillais? de l'Agence d'urbanisme de l'agglomération marseillaise, analyse à l'échelle des 111 quartiers marseillais l'origine de la population, et identifie les profils des individus selon leur nationalité actuelle ou à la naissance tels que disponibles dans les données INSEE.

12. $60 \%$ des élèves vivent dans des familles de quatre enfants et plus, jusqu'à 12 enfants par fratrie.

13. Ces caractéristiques rejoignent celles mises en évidence pour les collégiens issus de l'immigration, par Georges Felouzis, en 2003.

14. Unités pédagogiques des élèves allophones arrivants.

15. Cela ne signifie pas que les collégiens d'origine étrangère soient dans des dispositifs UPE2A, qui sont réservés aux allophones arrivant en France, mais qu'ils sont scolarisés dans des établissements qui accueillent ces dispositifs (nous ne disposons pas des données spécifiques relatives aux effectifs des élèves effectivement inscrits en dispositif UPE2A). 
16. Dans l'académie de Bordeaux, $10 \%$ des établissements scolarisent $26 \%$ des élèves allochtones, et $48 \%$ des élèves de ces établissements sont de milieu social défavorisé (Felouzis, Liot \& Perroton, 2005, p. 38).

17. Les limites des secteurs scolaires ne correspondant pas à celles des unités statistiques de l'INSEE (IRIS), un appariement direct des données issues des sources du Rectorat et de l'INSEE n'est pas possible (même si certains travaux s'y sont attelés, à partir de plusieurs opérations statistiques complexes - François et Poupeau, 2008). Aussi, nous utilisons séparément les données INSEE pour contextualiser (figure 1), et les données du Rectorat, géolocalisées (à l'adresse) pour l'ensemble des collégiens marseillais, afin de caractériser les secteurs scolaires et les collèges (figure 3).

18. Cet établissement a basé son projet sur l'accueil de tous : «Dans nos établissements vivent des personnes de religions, d'origines et de cultures différentes : c'est la richesse de Saint-Mauront. Loin des clivages de la société, cette richesse permet le respect des consciences et la rencontre de l'autre différent. [...] Les relations prennent appui sur l'altérité, chemin qui ouvre à la fraternité ", http://www.college.stm13.com/projet_peda/.

19. $26 \%$ d'immigrés dans le $1^{\text {er }}$ arrondissement et $23 \%$ dans le deuxième, soit près du double de la moyenne de Marseille (13\%) - données INSEE, 2012.

20. Bien que l'on parle en général d'évitement, nous préférons ici utiliser le terme de nonrespect, car les logiques de non-respect de la carte scolaire ne correspondent pas toujours à des logiques d'évitement, mais aussi à des logiques d'affectation (cf. infra).

21. Nous n'avons malheureusement pas pu obtenir les données (gérées en France par l'Inspection académique) relatives aux dérogations, dont on sait qu'à l'échelle nationale elles concernent environ 10 à $12 \%$ de la population scolaire (Inspection générale de l'éducation nationale, 2013).

22. Sections d'enseignement général et professionnel adapté. Elles constituent une forme de préorientation professionnelle des élèves en grande difficulté. On y trouve un public très largement issu de milieux défavorisés, et ces sections sont surtout concentrées dans les réseaux d'éducation prioritaire (13 établissements en disposent à Marseille, scolarisant $3 \%$ des collégiens).

23. Circulaire $\mathrm{n}^{\circ} 2002-100 \mathrm{du} 25$ avril 2002.

24. Collégiens scolarisés dans un établissement à l'étranger l'année précédente ; on suppose qu'ils sont pris en charge dans des UPE2A.

\section{RÉSUMÉS}

Alors que l'école affiche des objectifs de mixité sociale, la carte scolaire française tend à reporter les formes de la ségrégation sur les établissements scolaires des quartiers, en reproduisant les inégalités sociales. Pourtant, près de la moitié des collégiens marseillais échappent à la carte scolaire en la contournant. Les territoires scolaires, correspondant aux espaces de recrutement effectifs des établissements, sont-ils à même de réduire ces inégalités ? Par une analyse d'une base de données inédite à l'échelle des collégiens de la ville de Marseille, nous interrogeons les processus de ségrégation socio-spatiale et l'ethnicisation des territoires scolaires d'une commune pauvre, inégalitaire, et marquée par la présence de populations d'origine étrangère. Nos résultats montrent que les stratégies d'évitement des familles les plus favorisées vers les établissements privés, comme les orientations vers des établissements publics à l'offre spécifique, contribuent à renforcer les inégalités socio-ethniques des secteurs scolaires, et à spécialiser les territoires scolaires des établissements. 
In France, social mixing is one of the main objectives of school policies. The French school mapping system tends to reproduce urban socio-spatial segregation at school. Nonetheless, nearly half of the students in Marseille's middle schools avoid the school mapping system, attending other schools than the one in their neighbourhood they are supposed to attend. In which way are school territories, defined by the effective public of schools, able to reduce inequalities? The analysis of a school administration's database unfolds the processes of sociospatial segregation and ethnicization of school territories in Marseille, a poor, unequal and immigrants' city. The results indicate that strategies of avoidance of school sectors among upper class families who choose private schools, as well as school affectation's processes depending on specific school offer, both contribute to reinforce socio-ethnic inequalities at school, and to specialize school territories.

\section{INDEX}

Keywords : social geography, school inequalities, segregation, ethnicization, middle school, Marseille

Mots-clés : géographie sociale, inégalités scolaires, ségrégation, ethnicisation, collèges, Marseille

\section{AUTEURS}

\section{GWENAËLLE AUDREN}

Géographe, Fondation Aix Marseille Univ, CNRS, TELEMME, Aix-en-Provence, France, audrengwenaelle@yahoo.fr

\section{VIRGINIE BABY-COLLIN}

Professeure de géographie, Institut Universitaire de France, Aix Marseille Univ, CNRS, TELEMME, Aix-en-Provence, France, virginie.baby-collin@univ-amu.fr 